\title{
THE NATURE OF ACUTE PAIN ${ }^{\dagger}$
}

\section{P. A. FOSTER*}

As an anaesthetist I would say that you should not rassume that the anaesthetist is necessarily an expert on acute pain simply because this speciality controls usually successfully - part of the pain caused by the surgeon: The reason is that the very routine nature of the surgical assault encourages a narrow approach to the handling of pain relief which often, unfortunately, does not extend much beyond the operating theatre. The treatment of postoperative pain in fact of ten leaves much to be desired. To avoid the anaesthetist's close-up view of pain, l would like to paint for you a broader picture including some perspective and even a horizon.

To begin somewhere in the foreground, let us first state that the animal body stays in contact with and reacts to its environment by circuits in the nervous system we call reflex arcs. These are built according to the simple plan of -

RECEPTOR $\rightarrow$ SENSORY NERVE $\rightarrow$ CENTRALLY LOCATED SYNAPSE $\rightarrow$ MOTOR NERVE $\rightarrow$ EFFECTOR ORGAN.

Pain is part of a complex elaboration on this theme whereby an acutely received stimulus that exceeds a certain threshold must be perceived to be so unpleasant that immediate avoidance is demanded.

At this point may I emphasise that Acute Pain is an essential and valuable indicator for the body, which we should only ignore under special circumstances such as during surgery.

Chronic pain does not necessarily play such a valuable tunction.

The first step in the consideration of acute pain is to look at the pathways in the pain mechanism. This not only helps to understand the nature of pain but gives an insight into how to treat it. To re-emphasize the point already made, pain, in all its aspects, is an elaboration of the basic reflex arc which may be divided into -

DATA ACQUISITION $\rightarrow$ DATA PROCESSING $\rightarrow$ EXECUTIVE MOTOR PROGRAMMING.

We can look at these three phases separately and then see how they may interact.

There are a few of the simple facts about pain that need to be clearly understood.

There are two types of pain - a "fast pain", sometimes called the "first pain" that arises from the skin, localizes the site of injury, leads to the initial withdrawal reflex and does not outlast the stimulus. It is conducted along the $A \alpha$ group of fine myelinated

* Professor and Head, Department of Anaesthesia, University of Stellenbosch and Tygerberg Hospital.

† Paper read at Pain Symposium, preceding 13th National Council Meeting 23-27 April 1979. fibres, and is appreciated in the cerebral cortex after passage in the spinothalamic tracts.

Following this comes the "slow pain" or "real pain", the persistent pain that follows injury and leads to a different sort of reflex response - the guarding, rigidity or spasm that protects an injured part. The pathway here is along unmyelinated fibres which are slow conductors, hence the descriptive name. Appreciation is in subcortical brain areas subserved by the spinoreticulodiencephalic tracts.

"Real pain" pathways arise from skin and deeper tissue, and autonomic pain fibres mingle with the somatic fibre input which makes it possible to feel pain from deep organs referred to the surface of the body. The third spatial dimension of pain - depth - is often not as accurate as the surface location.

Perhaps the most important reason for the distinction between the two types of pain is because morphine is only effective in slow pain pathways. Thus morphine on its own cannot be an effective anaesthetic, since it does not influence "fast pain", but only pain from injury already sustained.

Pain is produced by four sorts of stimuli-

(a) Mechanical injury either to nerves or their endings;

(b) The thermal extremes of heat and cold;

(c) Electrical stimuli which directly fire sensory nerves and can produce pain without much injury;

(d) Chemical pain, produced by many substances either applied to tissue or liberated in tissue.

The first three are direct effects on ordinary nerve fibres or nerve endings, there being no specific pain receptors. Pain is the interpretation of the intensity and duration of a wide variety of stimuli carried along ordinary nerves.

Chemical pain is significant because it is also an indirectly produced pain secondary to tissue damage. It may be caused by the potassium liberated from injured cells, and by acid metabolic substances, or by the hormones of injury and inflammation. Substances such as histamine, bradykinins and prostaglandins are for us extremely important in acute pain because we have the chemical antagonists to use against them. Aspirin is a specific bradykinin antagonist and an inhibitor of prostaglandin synthesis. The recent introduction of intravenous aspirin into South Africa is thus an exciting new addition to our armamentarium against pain. So also, one should understand that oxygen lack, inadequate blood supply, venous congestion, swelling, are causes of pain that are treatable by simple physical means such as massage, positioning, mobilization, cold or an oxygen mask. Surely this is how pain should be ideally treated - at its source with physical means and 
specific drugs, rather than centrally with shotgun blasts from anaesthetists, morphine-like drugs and the like.

Over one aspect there is some confusion. Pain perception thresholds of nerves vary very little in people. Pain tolerance levels differ greatly between people and in the same person from time to time, but this is variation in interpretation or processing, not in the feeling or acquisition.

\section{PROCESSING THE INFORMATION}

To understand the difference between perception and tolerance, one must look to see how the information is processed objectively and subjectively.

Pain information, after being received and sorted when it enters the spinal cord, ascends to the brain along two main routes for processing - the first, one might say, for objective assessment, the second for subjective assessment. Already in this first sorting station in the spinal cord there is the blending of inputs from the somatic and visceral structures that can lead to referred pain and some of the abnormal pain syndromes.

For the objective appreciation of the painful stimulus, one must detect it accurately: Where is it? How long did it last? What is the context?

The subjective parhway - the spino-reticular pathway with its modulating circuits - is apparently more concerned with allotting significance to the stimulus as something harmful and to be avoided:

Arousal of brain activity for assessment and effective response.

Setting and detecting the threshold at which "pain" becomes painful

Interpreting the significance of the pain.

Deciding what to do.

It is suggested that there may be control mechanisms interacting between the two pathways. One of these runs from the brain back along the real pain pathway to a "gate", proposed by Melzack and Wall in 1965, which is present at the point of entry of the sensory nerve into the spinal cord in the substantia gelatinosa. There are other moderating pathways in the mid-brain itself that do not descend to spinal cord level. These are negative feedback or inhibitory patt ways of a type common in the organization of the central nervous system, which can reduce sensory input for various good reasons, such as greater priorities, directed attention, strong suggestion. We know what chemical transmitters are involved, and can mimic them with morphinelike drugs. The inhibition can be selective, so that a person may not react to severe threatening injury, but complain about non-significant and trivial pain. Thus one sees inhibition and disinhibition on the one hand of input, and on the other hand of interpretation.

Finally there is the appreciation of mental pain: danger, guilt, fear, grief, loss and memory, conceived rather than felt. Often the responses are similar. Undoubtedly pre-existing mental pain strongly influences the later perception of physical pain. A most significant brain region concerned with pain appreciation is the limbic area in which also emotion is appreciated.

\section{THE RESPONSE}

In looking at the responses to a stimulus that has been iudged painful, the motor side of the reflex arc is found to have many facets:

Vocalization: the warning call, the cry of submission or fear.

Violent muscular movement, or the inhibition of movement.

Secretion of adrenaline and from other glands.

Changes in the blood's biochemistry.

The emotional response.

I suppose it is a philosophical point as to whether the existing emotional state influences the significance that we read in pain, or whether it is the bringing together of the two judgements on the context of the pain and its significance as a threat that directs the pattern of the emotional response. Certainly the emo. tional response generated by mental pain or indifference determines how we feel physical pain. Probably this is of no great importance here as long as we remember the one most significant point, that the brain area for pain appreciation is inextricably intermingled with emotion. Because emotional response is the basis of the richness and complexity of the human experience pain becomes by its association, the most complex symptom we have to treat. Much of this complexity lies in the approach to chronic pain and is outside the scope of this discussion, but one must never forget:

that apprehension of pain makes its appreciation more intense;

that frequently apprehension is based on fear of an unknown and undefined experience, which we can reduce:

that strong emotion or motivation can also reducd the significance and the appreciation of pain. All our non-specific centrally acting pain relieving drugs are accompanied in their effects by psychical responses as well. Such psychical effects are usually pleasant - with these drugs addiction is a problem.

Looking forward to pain, either positively or negatively, can influence what one feels. So does looking back on the past experience. It is part of the cultural heritage of most civilizations to use pain to mould personalities, to establish behaviour patterns, to reinforce codes of morality, to discipline communities to conformity to arbitrary standards. How often is it the remembrance of unpleasant things past that make people toe the line.

If the significance of pain is so bound up with the future and the past, maybe we, the anaesthetists, have come upon a new approach to pain control. Two important properties of many of our new and potent drugs are those of strong amnesia coupled with tranquillization. These drugs are beginning to replace the classical anaesthetic drugs which have additional widespread effects in the body.

Do our new techniques with our new drugs perhaps expose the patient to the exquisite reality of present pain whilst at the same time eliminating any anxiety for what will happen in the next moment of time, and giving a calm forgetfulness of the pai । that has just passed? Will this become the new fonn of successfur anaesthesia - living only in an excruciating presen which one can never remember? Is pain a fear that what has happened will again happen?

How will we ever be able to find out?

\section{TREATING ACUTE PAIN}

It must be apparent from looking at acute pain mechanisms that there is a multitude of treatments. A purely anatomical approach will suggest various levels of attack from skin receptor up to the brain where the understanding of mechanisms is not so clear. On a nother approach level there are exciting possibilities to explore in controlling thresholds of pain, attitudes and our interpretation of the significance. We may modify the emotional response. We may also modify the motor response to pain and find that it influences our perception.

\section{The Sensory Nerve}

This has been blocked for many years by locally applied cold and later by the local anaesthetics, either at the nerve ending or along the nerve fibres as they run to the spinal cord. For as long, but on a less well 
understood basis, we have blocked the tissue hormones with aspirin. Now that we have so many more drugs to control the release or effects of tissue hormones we can achieve considerable pain relief by controlling the tissue response to trauma - swelling, inflammation and the liberation of chemicals from the damaged cell.

In our age of burgeoning pharmacology we sometimes overlook the value of treatments that restore normal function to an area of the body. Ischaemia causes pain; is there adequate oxygen supply or nutrient supply to the injured part? Disturbed function in one area may disturb another area to cause pain. This is a very large subject and in large measure it is what physiotherapy is about.

\section{The Spinal Cord}

Many of the most effective nerve blocks available to us are on the spinal cord after the first reception station of pain information. These spinal and extradural blocks that we do offer another possibility of blocking different types of nerves so that fast pain may be separated from slow pain and muscle power can be unaffected while we produce graded sensory block. It is valuable to be able to do this in the investigation of unusual pain syndromes, and to separate autonomic pain from somatic pain.

Autonomically active drugs are frequently overlooked in pain treatment. If you can relieve the spasm of an artery in migraine, or of the gut in the summer fruit season or of the uterus during menstruation, you relieve acute pain integrated at spinal cord level.

Other drugs reach the spinal cord in the blood stream; these the morphine-like drugs are tcdiy the most important. However, with new understanding of the synaptic transmitters involved in pain pathways in the spinal cord, new drugs will without doubt be developed.

\section{The Brain}

We have drugs that influence the objective response but do not serve as good analgesics, rather producing sleep, by acting apparently only on the cerebral cortex.

Many of the drugs used in psychiatry have a place in treating pain which is not unexpected if you think of much mental disease as uncontrolled mental pain.

The euphoriant drugs such as cocaine, benzedrine and methedrine, which we are not allowed to use, have profound effects on pain perception, making it less important, more bearable.

Antidepressant drugs likewise have an established place in treating chronic pain; unfortunately most are too slow acting to be of use with acute pain. If one looks for a fast acting antidepressant drug one comes back to morphine which has been used from time immemorial for this purpose.

Amnesic drugs, whilst they may not appear from the patient's response to bring pain relief, are used nevertheless. "Twilight sleep" with scopolamine for "painless childbirth" is an example.

What should be more fully explored is the use of combinations of drugs to suit individuals. Where morphine is insufficient we frequently combine it with tranquillizers (diazepam, lorazepam, droperidol) or antidepressants such as chlorpromazine and achieve a better effect. Still better is to combine such a combination with one of the peripherally acting drugs, such as intravenous aspirin.

Anaesthetics probably act in a morphine-like way and also affect fast pain perception by producing sleep.

\section{The Response}

How much does the adrenaline secretion, or the spasm of injured muscle influence pain perception? Certainly both are powerful arousal mechanisms of the reticular system which is associated with real pain perception. Whilst neither curare nor beta adrenergic blocking drugs are regarded as analgesics, they are used in drug combinations which makes it look as if they might have analgesic effects. Anaesthetists don't often think about this.

Surely the greatest error we make in treating pain is to believe that a single approach must be effective. And the second error arises from our inability to measure pain in someone else so that we deceive ourselves that our treatment sometimes works better than it in fact does.

If we regard pain as a complex response with pathways at all levels from the individual cell to the most highly integrated mental function, then we may better control it. 